Meta

Journal des tradlucteurs

Translators' Journal

\title{
Procédés de formation et matrices terminogéniques en terminologie des systèmes experts
}

\section{Monique C. Cormier et Louis-Paul Rioux}

Volume 36, numéro 1, mars 1991

La terminologie dans le monde : orientations et recherches

URI : https://id.erudit.org/iderudit/002399ar

DOI : https://doi.org/10.7202/002399ar

Aller au sommaire du numéro

Éditeur(s)

Les Presses de l'Université de Montréal

ISSN

0026-0452 (imprimé)

Découvrir la revue

Citer cet article

Cormier, M. C. \& Rioux, L.-P. (1991). Procédés de formation et matrices terminogéniques en terminologie des systèmes experts. Meta, 36(1), 248-268. https://doi.org/10.7202/002399ar d'utilisation que vous pouvez consulter en ligne. 


\title{
PROCÉDÉS DE FORMATION ET \\ MATRICES TERMINOGÉNIQUES EN TERMINOLOGIE DES SYSTEMES EXPERTS*
}

\author{
Monique C. Cormier et Louis-Paul RiouX \\ Université de Montréal, Montréal, Canada
}

Les systèmes experts constituent l'une des avenues les plus prometteuses en intelligence artificielle. Bien que, à ce jour, il y ait eu un certain nombre d'études terminologiques ayant pour objet l'intelligence artificielle (Boulanger 1988; Pavel 1986; Pavel 1988) ou certaines de ses applications (notamment la productique et la traduction automatique [Coiffet 1986; Kooi 1989]), à notre connaissance, aucune des études publiées ne traite des procédés de formation dans le domaine particulier des systèmes experts.

L'objet du présent article est de rendre compte des résultats d'une recherche sur l'identification des procédés de formation des termes dans le domaine des systèmes experts et des matrices terminogéniques les plus productives.

Le corpus sur lequel a porté l'analyse compte 625 termes; il provient du dépouillement de divers ouvrages spécialisés dans le domaine de l'intelligence artificielle, et plus spécifiquement dans celui des systèmes experts ( $c f$. Annexe 1). Il ne se prétend pas exhaustif, mais il semble représentatif de l'ensemble du technolecte français de ce domaine. En effet, il contient les variantes orthographiques de nombreux termes et quelques emprunts sous forme de xénotermes (Boulanger 1988:4).

Une première constatation: le corpus étudié contient beaucoup plus de termes avec extension (ou multitermes; ex. : fait incertain) que de termes seuls (ou monotermes; ex.: heuristique). Ainsi, sur un total de 625 termes, on trouve 479 multitermes (y compris les sigles), soit $76,6 \%$ du corpus, et 135 monotermes ( $21,6 \%$ ); quant aux 11 termes restants $(1,8 \%)$, il s'agit de xénotermes incluant des mono et des multitermes, de même que des sigles.

Les principaux procédés de formation, autant en terminologie qu'en lexicologie, sont la dérivation et la composition. Nous avons déjà une bonne indication de l'importance de ce dernier procédé, puisque plus de $75 \%$ des termes du corpus sont des multitermes. Cependant, il peut arriver que la dérivation ait lieu au sein d'un composé. Il n'y a donc pas de clivage net entre cas de dérivation et cas de composition.

\section{COMPOSITION}

Les termes formés par composition constituent la plus grande proportion du corpus. Nous les avons divisés en dix catégories principales: 
TABLEAU 1. Composés

\begin{tabular}{|c|c|c|c|}
\hline & TYPE & NOMBRE & $\%$ \\
\hline $\mathbf{1}$ & N adj... & 161 & 33,6 \\
\hline $\mathbf{2}$ & N adv $\ldots$ & 10 & 2,1 \\
\hline $\mathbf{3}$ & N conj... & 3 & 0,6 \\
\hline $\mathbf{4}$ & N N ... & 37 & 7,9 \\
\hline $\mathbf{5}$ & N prep ... & 214 & 44,7 \\
\hline $\mathbf{6}$ & N V ... & 6 & 1,3 \\
\hline $\mathbf{7}$ & N X ... & 11 & 2,1 \\
\hline $\mathbf{8}$ & adv-N... & 6 & 1,3 \\
\hline $\mathbf{9}$ & X-N & 13 & 2,7 \\
\hline $\mathbf{1 0}$ & SIGLES & 18 & 3,8 \\
\hline
\end{tabular}

Dans ce tableau, une expression du type $\mathbf{N}$ adj... désigne un terme composé d'un nom suivi d'un adjectif, et facultativement suivi d'un ou de plusieurs autres composants. Selon ce relevé, deux procédés de formation sont très productifs : $\mathbf{N}$ prep... et $\mathbf{N}$ adj...; cing le sont moyennement: $\mathbf{N}$ adv..., $\mathbf{N} \mathbf{N}$..., $\mathbf{N} \mathbf{X} \ldots, \mathbf{X} \mathbf{N}$ et la siglaison, tandis que les trois autres procédés le sont peu ou pas. Nous procéderons à l'analyse de chacun d'entre eux par ordre décroissant de productivité, sauf pour la siglaison, qui sera traitée à la toute fin de cette section.

N PREP...

Les composés formés d'un nom suivi d'une préposition et d'un ou de plusieurs autres éléments donnent lieu à de multiples matrices terminogéniques (plus de 20). Cependant, la plupart de ces matrices ne génèrent qu'un ou deux termes. De plus, comme on le verra, il est possible de considérer que certaines d'entre elles sont, à certains égards, des variantes d'une matrice plus universelle.

C'est sans surprise que l'on constate la forte proportion de $\mathbf{N}$ prep $\mathbf{N}$ parmi ce sous-ensemble (128 termes sur un total de 214 , soit près de $60 \%$, et $27 \%$ de l'ensemble des multitermes), compte tenu de la fréquence de cette matrice dans la plupart, sinon dans tous les technolectes français. Selon la préposition employée, on retrouve la répartition suivante: 
TABLEAU 2. $\mathbf{N}$ prep $\mathbf{N}$

\begin{tabular}{|c|c|c|}
\hline PRÉPOSITION & NOMBRE & $\%$ \\
\hline d' & 27 & 21,1 \\
\hline de & 76 & 59,4 \\
\hline des & 11 & 8,6 \\
\hline du & 1 & 0,8 \\
\hline en & 6 & 4,7 \\
\hline par & 7 & 5,5 \\
\hline
\end{tabular}

Une majorité de termes comporte la préposition «de» et, dans une moindre mesure, sa variante élidée «d'»: base de faits, fragment de savoir, jeu d' essai, moteur d' inférence, etc. Suivent les termes en «des», espace des solutions, représentation des connaissances, etc., ceux qui contiennent «par», programmation par apprentissage, règle par défaut, etc., puis ceux avec «en», mise en correspondance, recherche en largeur, etc. Quant à la préposition «du», elle n'est représentée que par un seul terme, explication du raisonnement. Fait à noter: l'absence totale de composés en «à» ou «au» pour cette matrice.

Toujours en suivant l'ordre décroissant de productivité, on obtient les matrices suivantes:

- $\mathbf{N}$ prep $\mathbf{N}$ adj (17 termes): ils se décomposent à leur tour en $15 \mathbf{N}$ prep ( $\mathbf{N}$ adj) (atelier de génie cognitif, hypothèse du monde ouvert, représentation par objets structurés, système à tableau noir, etc.) et deux ( $\mathbf{N}$ prep $\mathbf{N}$ ) adj (base de données déductive et moteur d' inférences $n u$ ).

- N prep det $\mathbf{N}$ (15 termes) : neuf dont le déterminant est «l'» ou «la» (document de la connaissance, traitement de l'incertain, transfert de l'expertise, etc.) et six avec le déterminant «un» ou «une» (portée d'une variable, sémantique d'un nœud, etc.).

- $\mathbf{N}$ prep $\mathbf{N}$ prep $\mathbf{N}$ (15 termes) : $13 \mathbf{N}$ prep (N prep $\mathbf{N}$ ) (cycle du moteur d'inférences, outil d'aide au développement, représentation par règles de production, système à base de connaissances, etc.) et deux (N prep $\mathbf{N}$ ) prep $\mathbf{N}$ (mise à jour de connaissances et outil de développement de $S E$ ).

- $\mathbf{N}$ prep (N prep det $\mathbf{N})$ ( 7 termes) : logiciel d' aide à la décision, module d' aide à la validation, module de récupération de l'expertise, outil de maintien de la cohérence, etc. En fait, sur un total de sept termes, cinq contiennent la suite «...aide à la...».

- N prep N X (5 termes) où «prep N» se traduit toujours par «d'ordre»: logique d'ordre 0, moteur d'ordre 0, moteur d'ordre 1 , moteur d'ordre 2 , moteur d'ordre $0+$.

- N prep adj N (5 termes): connaissance de bon sens, logique à plusieurs valeurs, logique du premier ordre, prédicat du premier ordre, système de deuxième génération.

- $\mathbf{N}$ prep $\mathbf{N}$ adv (4 termes) débutant tous par «recherche»: recherche avec retour arrière, recherche du meilleur d'abord, recherche en largeur d'abord, recherche en profondeur d'abord.

Les autres matrices génèrent trois termes ou moins:

- $\mathbf{N}$ prep $\mathbf{N}$ prep $\mathbf{N}$ adj: logiciel de développement de systèmes experts, outil de développement de systèmes experts, système à base de connaissances interactif. 
- $\mathbf{N}$ prep $\mathbf{N}$ prep $\mathbf{N}$ prep $\mathbf{N}$ : cycle de base du moteur d'inférence, module d'aide au transfert d'expertise.

- $\mathbf{N}$ prep $\mathbf{N}$ prep det $\mathbf{N}$ prep $\mathbf{N}$ : outil d'aide à l'acquisition de connaissances, système d'aide à la prise de décision.

- $\mathbf{N}$ prep det $\mathbf{N}$ prep $\mathbf{N}$ : aide à la prise de décision, graphe de l'espace des états.

- $\mathbf{N}$ prep $\mathbf{N}-\mathbf{N}$ : opération d' unification-instanciation.

- $\mathbf{N}$ prep $\mathbf{N}$ prep $\mathbf{N}$ prep det $\mathbf{N}$ : module d'aide au maintien de la cohérence.

- $\mathbf{N}$ prep $\mathbf{N}$ prep $\mathbf{N}$ adj conj adj: machine à inférence à architectures parallèles et intégrées.

- $\mathbf{N}$ prep $\mathbf{N}$ prep det $\mathbf{N}$ adj : système d'aide à la prescription médicale.

-- $\mathbf{N}$ (prep $\mathbf{N}$ conj prep $\mathbf{N}$ ) : interpréteur (de faits et de règles).

-- $\mathbf{N}$ prep $\mathbf{N}$ conj prep $\mathbf{N}$ prep $\mathbf{N}$ : cycle d'acquisition et de rafraîchissement des données.

- $\mathbf{N}$ prep $\mathbf{N}$ adj conj prep $\mathbf{N}$ prep $\mathbf{N}$ : validation en temps réel et à pied d'œuvre.

-- $\mathbf{N}$ prep $\mathbf{V}$ adj $\mathbf{N}$ : capacité d'atteindre toute conclusion.

-- $\mathbf{N}$ prep $\mathbf{V}$ prep adj $\mathbf{N}$ prep $\mathbf{N}$ adj: capacité de discriminer parmi tout ensemble de conclusions possibles.

Ces deux dernières matrices ( $\mathbf{N}$ prep V...) sont à tout le moins accidentelles; les deux termes qui leur correspondent auraient été rejetés, s'ils n'avaient fait l'objet d'une siglaison (CAC pour capacité d'atteindre toute conclusion et $C D C$ pour capacité de discriminer parmi tout ensemble de conclusions possibles); cette particularité a mis en évidence l'existence de ce type de terme ou, à tout le moins, a légitimé sa présence dans le corpus.

De la même façon, les quatre matrices contenant une conjonction sont elles aussi improductives, puisque circonstancielles : la puissance de création de ce type de matrices est illimitée, de par la propriété de la conjonction d'ajouter aux termes autant d'extensions qu'il est possible d'imaginer. En conséquence, la probabilité de retrouver la même matrice pour deux termes ainsi arbitrairement formés serait très mince. Mais, bien qu'elles soient improductives, ces matrices génèrent des termes parmi les plus complexes et les plus intéressants: validation en temps réel et à pied d' œuvre, machine à inférence à architectures parallèles et intégrées, cycle d'acquisition et de rafraîchissement des données.

Par ailleurs, au sein de cet ensemble de 21 matrices, il est possible de procéder à un certain nombre de recoupements. Certaines, très peu productives, peuvent être rattachées à d'autres plus importantes.

Ainsi, d'une manière générale, lorsqu'un déterminant est inséré dans une matrice n'en possédant pas, cette nouvelle matrice est indéniablement apparentée à la matrice de départ. La présence du déterminant se justifie alors uniquement par un besoin d'euphonie (comparer module de récupération de l' expertise et «module de récupération d'expertise», compte tenu que transfert d'expertise et transfert de l'expertise se retrouvent concurremment dans le corpus), ou afin d'assurer au terme une construction syntaxique adéquate (système d'aide à la conception plutôt que *système d'aide à conception; ingénieur de la connaissance et *ingénieur de connaissance, etc).

Vus d'un autre angle, les termes employant les groupes prépositionnels «de l'» ou «de la» (raisonnement par l'absurde, traitement de l'incertain, document de la connaissance, etc.) sont à rapprocher des termes comportant la préposition «du» (qui équivaut, rappelons-le, au groupe «de le»). On remarque également qu'ils font tous référence à des concepts génériques: la connaissance/une ou des connaissances, l'incertitude/une incertitude (traitement de l'incertitude), l'expertise/une expertise 
(transfert de l'expertise); ou une conversion adj $\rightarrow \mathrm{n}:$ logique de l'incertain, raisonnement par l'absurde, etc.

Par ailleurs, considérons le terme opération d'unification-instanciation qui a été analysé $\mathbf{N}$ prep $\mathbf{N}-\mathbf{N}$. Il répond davantage à la matrice $\mathbf{N}$ prep $\mathbf{N}$, puisqu'il est possible de considérer que les deux derniers éléments, reliés par un trait d'union, constituent une unité nominale autonome.

Considérons d'autre part les unités $S E$ et $I A$, respectivement sigles des termes système expert et intelligence artificielle; on les retrouve dans les composés système d'IA et outil de développement de SE. Tous deux ont été traités comme des monotermes entrant eux-mêmes dans la formation de nouveaux termes; dans le cas présent, les deux composés générés répondent donc aux matrices $\mathbf{N}$ prep $\mathbf{N}$ et $\mathbf{N}$ prep $\mathbf{N}$ prep $\mathbf{N}$. D'ailleurs, les équivalents non abrégés de ces deux termes sont également présents dans le corpus: système d'intelligence artificielle et outil de développement de systèmes experts.

$\mathrm{Ce}$ cas particulier de formation a permis de mettre au jour un phénomène omniprésent au sein du corpus, soit la composition au second degré, c'est-à-dire le procédé par lequel un terme composé présent dans le corpus génère à son tour un ou plusieurs autres composés.

Ainsi, système expert et intelligence artificielle, mais également plusieurs autres termes, contribuent à la formation de nouveaux termes: base de connaissances (système à base de connaissances, système à base de connaissances interactif), moteur d'inférences (cycle du moteur d'inférences, cycle de base du moteur d'inférence, moteur d'inférences $n u$ ), outil de développement (outil de développement de SE, outil de développement de systèmes experts), prise de décision (aide à la prise de décision, système de prise de décision, système d'aide à la prise de décision), règle de production (programmation en règles de production, représentation par règles de production, système à règles de production, système expert à règles de production) et, dans une moindre mesure, acquisition de connaissances (outil d'aide à l'acquisition de connaissances), base de données (base de données déductive), espace des états (graphe de l'espace des états), génie cognitif (atelier de génie cognitif), génie logiciel (atelier de génie logiciel), objets structurés (représentation par objets structurés), tableau noir (système à tableau noir) et transfert d' expertise (module d'aide au transfert d'expertise).

Par ailleurs, on note dans certains termes la présence de syntagmes de la langue générale qui, bien que n'apparaissant pas dans le corpus, n'en sont pas moins figés, tels «bon sens» (connaissance de bon sens), "sens commun» (raisonnement de sens commun), «temps réel» et «à pied d'œuvre» (validation en temps réel et à pied d' auvre).

Qu'adviendrait-il si l'on décidait d'analyser ces composés en partant de l'hypothèse que, au second degré, les termes les formant étaient eux-mêmes des unités? Pour prendre un exemple, le terme représentation par objets structurés serait analysé $\mathbf{N}$ prep $\mathbf{N}$, plutôt que $\mathbf{N}$ prep $\mathbf{N}$ adj, puisque objet structuré est un terme du corpus. Première conséquence : la précédente liste de matrices avec leurs exemples devrait être réaménagée. Deuxième conséquence : certaines matrices deviendraient inutiles.

Ainsi, les deux termes de la matrice $\mathbf{N}$ prep (det $\mathbf{N}$ prep $\mathbf{N}$ ), aide à la prise de décision et graphe de l'espace des états, devraient selon notre analyse être portés à la liste des $\mathbf{N}$ prep det $\mathbf{N}$.

Une autre matrice ( $\mathbf{N}$ prep $\mathbf{N}$ prep $\mathbf{N}$ adj) engendre trois termes pouvant être redistribués dans trois autres matrices, rendant la première inutile. Ainsi, logiciel de développement de systèmes experts serait ajouté à la liste des $\mathbf{N}$ prep $\mathbf{N}$ prep $\mathbf{N}$, et outil de développement de systèmes experts enrichirait les $\mathbf{N}$ prep $\mathbf{N}$, puisque outil de développement et système expert sont tous deux des termes autonomes du corpus. Quant au 
troisième terme, système à base de connaissances interactif, il se rangerait du côté des ( N prep N) adj.

Toutefois, en relocalisant ce dernier terme avec les ( $\mathbf{N}$ prep $\mathbf{N}$ ) adj, la présence de cette matrice devient incontournable, même si les deux termes qui la prennent comme modèle (base de données déductive et moteur d'inférences $n u$ ) sont eux-mêmes des $\mathbf{N}$ $\mathbf{a d j}$, d'après nos critères. De plus, la majorité des 15 termes en $\mathbf{N}$ prep $(\mathbf{N}$ adj) sont à classer parmi les $\mathbf{N}$ prep $\mathbf{N}$ : atelier de génie cognitif, fait à valeurs multiples, générateur de systèmes experts, ingénieur en intelligence artificielle, représentation par objets structurés, système à tableau noir, etc. Cependant, quelques autres termes n'entrent pas dans ce schéma (hypothèse du monde clos, machine à intelligence spécialisée, raisonnement de type monotone, etc.); par conséquent, la matrice $\mathbf{N}$ prep ( $\mathbf{N}$ adj) demeure nécessaire.

Les $\mathbf{N}$ prep $(\mathbf{N}$ prep $\mathbf{N})$ présentent la même difficulté, puisque près de la moitié des 13 termes de cette matrice sont à classer avec les $\mathbf{N}$ prep $\mathbf{N}$ (cycle du moteur d' inférences, programmation en règles de production, système à base de connaissances, système de prise de décision, etc.), mais puisque les termes restants ne répondent pas à cette analyse (ex.: capteur d'acquisition des données, outil d'aide au développement, problème du cadre de référence, programmation à base d'objets, etc.), cette matrice doit également être conservée.

Un cas encore plus subtil se présente: les deux termes formés d'après la matrice $\mathbf{N}$ prep $\mathbf{N}$ prep $\mathbf{N}$ prep $\mathbf{N}$ sont, selon notre interprétation, des $\mathbf{N}$ prep $\mathbf{N}$ prep $\mathbf{N}$ (cycle de base du moteur d'inférence, module d'aide au transfert d'expertise); de même, les deux termes de la matrice $\mathbf{N}$ prep $\mathbf{N}$ prep det $\mathbf{N}$ prep $\mathbf{N}$ (outil d'aide à l'acquisition de connaissances et système d'aide à la prise de décision) répondent plutôt à la matrice $\mathbf{N}$ prep ( $\mathbf{N}$ prep det $\mathbf{N}$ ). Cependant, il existe au moins un terme (module d'aide au maintien de la cohérence) qui n'est pas issu d'une composition au second degré, et dont la matrice, $\mathbf{N}$ prep $\mathbf{N}$ prep $\mathbf{N}$ prep det $\mathbf{N}$, correspond à une variante de la matrice principale $\mathbf{N}$ prep $\mathbf{N}$ prep N prep N. Dans ces conditions, il n'est plus possible d'éliminer cette dernière.

Par contre, une dernière matrice deviendrait redondante: $\mathbf{N}$ prep $\mathbf{N}$ adj conj prep $\mathbf{N}$ prep $\mathbf{N}$ (validation en temps réel et à pied d' euvre); «temps réel» et «à pied d'œuvre» étant figés, la matrice de ce terme serait plutôt $\mathbf{N}$ prep $\mathbf{N}$ conj $\mathbf{j}$ prep $\mathbf{N}$, qui correspond à celle du terme interpréteur (de faits et de règles).

Il n'y aurait donc en définitive que quatre matrices sur un total de 21 qui seraient éliminées: $\mathbf{N}$ prep $(\operatorname{det} \mathbf{N}$ prep $\mathbf{N}), \mathbf{N}$ prep $\mathbf{N}$ prep $\mathbf{N}$ adj, $\mathbf{N}$ prep $\mathbf{N}$ adj conj prep $\mathbf{N}$ prep $\mathbf{N}$, de même que $\mathbf{N}$ prep $\mathbf{N}-\mathbf{N}$ (opération d' unification-instanciation) qui, on l'a vu, est un cas particulier de $\mathbf{N}$ prep $\mathbf{N}$. Ce résultat n'est pas très significatif, mais il reste à vérifier l'effet de ce phénomène de composition au second degré sur les autres procédés de composition.

N ADJ...

Le type de composés $\mathbf{N}$ adj, avec ou sans extension, produit de nombreux termes: 161 composés, soit le tiers de l'ensemble des multitermes; seule la matrice principale $\mathbf{N}$ adj est très productive (141 termes, ou 87,6\% de l'ensemble des composés $\mathbf{N}$ adj...). Il existe huit autres matrices, mais parmi celles-ci, plusieurs deviennent inutiles si l'on considère la composition au second degré. Ce phénomène touche particulièrement les formes où apparaît le terme système expert.

- $\mathbf{N}$ adj adj adj : système expert universel vide (plutôt $\mathbf{N}$ adj $\mathbf{a d j}$ )

- $\mathbf{N}$ adj $\mathbf{N}$ adj: système expert temps réel $(\mathbf{N} \mathbf{N})$

- $\mathbf{N}$ adj prep adj $\mathbf{N}$ : système expert de deuxième génération ( $\mathbf{N}$ prep adj $\mathbf{N}$ )

- $\mathbf{N}$ adj prep $\mathbf{N}$ prep $\mathbf{N}$ : réseau sémantique à propagation de marqueurs $(\mathbf{N}$ prep $\mathbf{N}$ prep $\mathbf{N}$ ), système expert à règles de production (N prep $\mathbf{N})$ 
- $\mathbf{N}$ adj X-N : système expert multi-niveaux $(\mathbf{N} \mathbf{X}-\mathbf{N})$

Il reste alors véritablement quatre matrices :

- $\mathbf{N}$ adj (141 termes) : calcul parallèle, graphe simple, mode fermé, processeur symbolique, valeur-possible, etc.

- $\mathbf{N}$ adj adj (9 termes): composant logiciel réutilisable, formule logique cohérente, formule logique composée, moteur nu élémentaire; les cinq autres composés comportent le terme système expert et sont donc à rattacher à la matrice $\mathbf{N}$ adj : système expert auxiliaire, système expert diagnosticien, système expert industriel, système expert nu et système expert vide.

- $\mathbf{N}$ adj prep $\mathbf{N}$ (4 termes): résolveur général de problèmes, tri binaire par arbre, type abstrait de données; le quatrième terme, système expert à objets, est du type $\mathbf{N}$ prep $\mathbf{N}$.

- $\mathbf{N}$ adj prep $\mathbf{N}$ prep det $\mathbf{N}$ (1 terme) : système interactif d'aide à la décision.

Précisons au passage que le terme système expert est relativement ambigu. Expert est-il nom ou adjectif? S'il était nom, il s'agirait d'une apposition, soit un système se comportant en expert, ou plus justement un système reproduisant le comportement, le raisonnement d'un expert (d'un domaine précis et restreint). Mais si expert était adjectif, le sens serait conservé, puisque le terme serait interprété: 'système ayant les capacités d'un expert'; de plus, si l'on remplace «système» par un autre terme, par exemple «machine», il serait probablement plus acceptable d'écrire «machine experte» que «machine expert», c'est-à-dire qu'il y aurait accord en genre, signe de la présence d'un adjectif.

Par ailleurs, un terme de la matrice $\mathbf{N}$ adj comporte un trait d'union (valeurpossible), situation inhabituelle pour ce type de composés, puisque le trait d'union est davantage le fait de l'apposition, cas fréquent dans les composés $\mathbf{N} \mathbf{N}$.

N N...

Les composés $\mathbf{N} \mathbf{N}$... sont moyennement productifs (37 termes, ou 7,7\% du total des multitermes); mais, à elle seule, la matrice $\mathbf{N} \mathbf{N}$ génère 29 termes, notamment fonction membre, ingénieur IA, langage objets, stratégie essai, noeud-ancêtre, robotiquelaction, etc.

Trois autres matrices produisent les huit termes restants; l'une d'elles, $\mathbf{N} \mathbf{N}$ adj, doit se lire en réalité $\mathbf{N} \mathbf{N}$, puisque les deux termes qu'elle génère contiennent le syntagme «temps réel»: $S E$ temps réel et système temps réel. Une deuxième, $\mathbf{N} \mathbf{N}-\mathbf{N}$, qui produit cinq termes, s'apparente à celle qui a déjà été rencontrée lors de l'étude des $\mathbf{N}$ prep..., à savoir $\mathbf{N}$ prep $\mathbf{N}-\mathbf{N}$, qui avait été analysée $\mathbf{N}$ prep $\mathbf{N}$. Par conséquent, nous analyserons celle-ci $\mathbf{N} \mathbf{N}$, compte tenu de la présence du trait d'union. Notons pour ces derniers termes la fréquence de la suite «alpha-bêta»: algorithme alpha-bêta, analyse fins-moyens, procédé alpha-bêta, procédure alpha-bêta et processus producteur-consommateur. Quant à la dernière matrice, $\mathbf{N} \mathbf{N}$ prep $\mathbf{N}$ adv, elle produit un seul terme: stratégie essai avec retour arrière; stratégie essai et retour arrière étant constituants autonomes du corpus, la matrice devient $\mathbf{N}$ prep $\mathbf{N}$.

Si l'on revient aux termes de la matrice $\mathbf{N} \mathbf{N}$, il est important de mentionner que, pour plusieurs d'entre eux, il n'est pas toujours évident que le second élément soit un nom. Ainsi, pour interface expert, module expert ou utilisateur expert, on serait tenté de voir en expert un adjectif (tout comme pour système expert, voir plus haut), mais la présence dans le corpus des termes interface utilisateur et utilisateur opérateur vient tout remettre en question, car pour ces derniers, l'accent est mis sur la fonction et non sur la qualité rattachée à cette fonction. De plus, dans le cas de interface expert, on parle de l'interface pour l'expert et non pas de l'interface ayant les capacités d'un expert. Et, de toute façon, le terme interface est féminin. 
Noud descendant est également ambigu, mais par le contexte, il devient clair que descendant est ici synonyme de fils, et ne signifie pas 'vers le bas'. Enfin, pour le terme objet modèle, il serait possible de discuter longuement, mais il semble que, d'après le contexte, il ne s'agisse pas d'un objet «idéal» ou «sans défaut», mais bien d'un objet ayant la fonction de modèle, pris dans le sens de 'prototype'.

Mais lorsque le composé $\mathbf{N} \mathbf{N}$ comporte un trait d'union, il n'y a pas de doute possible, il s'agit bien de deux noms juxtaposés, et le concept de mot-tandem (Pavel 1989b: 348) prend tout son sens. Les six composés de cette sous-matrice $\mathbf{N}-\mathbf{N}$ débutent tous par le nom nœud, à l'exception d'un terme: nœud-ancêtre, nœud-but, nœud-feuille, noud-racine, noud-solution et forme-objet. De plus, deux autres composés $\mathbf{N} \mathbf{N}$ voient leurs éléments séparés par un trait oblique : robotiquelaction et robotique/perception.

Bien entendu, plusieurs des autres termes de la matrice $\mathbf{N} \mathbf{N}$ ne possédant pas de trait d'union sont également des mots-tandems; ce sont principalement les autres termes de la série «nœud»: nœud descendant, nœud parent et nœud père. De même que langage acteurs, machine multiprocesseurs, mode exploitation, procédure "démon», programmation objet, etc. Notons au passage la fréquence des termes ayant le nom objet en apposition: fonctionnalité objet, langage objets et programmation objet, tous issus de la troncation de la construction «orienté objets», elle-même héritée de l'anglais («object-oriented» [Quirion 1989: 44]).

Enfin, il est intéressant de s'interroger sur deux composés de cette matrice, qui sont peut-être davantage des $\mathbf{N}$ prep $\mathbf{N}$, à savoir mode développement et mode exploitation, qui pourraient aussi bien se lire «mode de développement» et «mode d'exploitation», sans que le sens soit modifié. Il s'agit sans doute d'une manifestation du calque de l'anglais, mais il est difficile de ne pas voir également pour ces termes une certaine usure de la préposition «de», qui, à force d'indiquer toutes les relations possibles entre constituants, en vient à ne plus rien indiquer du tout (Clas 1988: 224).

$\mathrm{X}-\mathrm{N}$

Le procédé de formation $\mathbf{X}-\mathbf{N}$ est à la limite de la composition, plus justement à l'intersection de la dérivation et de la composition. Il s'agit en fait de termes crés par préfixation d'un élément autonome, à un terme déjà présent dans le corpus. C'est le caractère d'autonomie de ces préformants qui peut justifier la présence de ce procédé au sein des composés. Par ailleurs, il n'existe qu'une seule matrice, soit simplement X-N; autrement dit, le corpus ne comporte aucune forme avec extension pour ce type particulier de composés.

Les termes de la matrice X-N sont au nombre de 13, et six d'entre eux sont formés à partir de l'élément «méta», lié ou non au terme par un trait d'union: méta-classe, métaconnaissance, méta-règle, métaconnaissance, métalangage et métaraisonnement. L'élément «méta», de plus en plus utilisé dans les technosciences, bénéficie d'une réelle autonomie puisque, dans le corpus, on retrouve le terme niveau méta. Originellement, cet élément signifiait 'après' («métacarpe», partie du squelette de la main venant après le carpe) (Lurquin 1982: 2); toutefois, par glissement de sens, principalement sous l'influence du terme «métaphysique» (dans les œuvres d'Aristote, connaissance traitée après la physique) désignant aujourd'hui une réflexion abstraite transcendant l'ordre sensible, «méta» signifie dans de multiples termes techniques contemporains 'qui dépasse, qui englobe' (ibid): métalangage, langage pour parler du langage, métaconnaissance, connaissance sur les connaissances, méta-règle, règle d'utilisation des règles, etc.

Deux autres termes emploient l'élément «hyper», 'au-dessus, au-delà' : hyperarc et hypergraphe. Trois termes contiennent l'élément «pré», 'en avant': précondition, 
préordre et présystème, tandis qu'un autre terme utilise «mi», 'moitié': mi-ordre. Quant au dernier terme, $k$-connecteur, il s'agit d'un cas limite puisque l'élément, bien qu'autonome (une lettre de l'alphabet), n'est pas un formant au même titre que les précédents. On ne peut, dans ces conditions, parler de préfixation.

N X...

Onze termes répartis sur quatre matrices sont générés par la combinaison d'un nom suivi d'un élément autonome non lexicalisé (chiffre, lettre, abréviation) ou formant, ce dernier étant facultativement relié à un autre élément lexicalisé :

- N X (3 termes): langage $C$, moteur 0 , niveau méta.

- $\mathbf{N ~ X - a d j ~ ( 4 ~ t e r m e s ) : ~ l o g i q u e ~ m u l t i v a l e n t e , ~ l o g i q u e ~ m u l t i - v a l u e ́ e ~ ( e t ~ s a ~ v a r i a n t e ~ l o g i q u e ~}$ multivaluée), représentation multi-modale.

- N X-N (3 termes): héritage inter-objets, système multi-agents, système multi-experts.

- N X-X (1 terme): arbre «min-max».

On peut constater qu'à l'instar des termes de la matrice $\mathbf{X}-\mathbf{N}$, ceux qui sont générés par les matrices $\mathbf{N} \mathbf{X}$-adj et $\mathbf{N} \mathbf{X}-\mathbf{N}$ sont des cas mixtes de composition et de dérivation, puisque les éléments «X» de ces termes sont des préformants du français, issus du latin («inter», 'entre', et «multi», 'beaucoup').

Cependant, leur présence est davantage justifiée au sein des composés, car l'adjonction du formant crée un tout indissociable; ainsi, en omettant l'élément formant dans le terme héritage inter-objets, la résultante («héritage objets») est incohérente. Tous les autres termes étudiés comportent le formant «multi»; de la même façon, le retrait de celui-ci peut conduire à des contresens ou des formations fautives: *logique valente, «système agents». Pour leur part, les termes «représentation modale» et «logique valuée» auraient une portée moins signifiante et seraient près du pléonasme. Une seule exception: système multi-experts, qui deviendrait simplement système expert.

Toutefois, l'unité formée à partir du préformant acquiert une certaine autonomie et, bien qu'on ne retrouve pas tels quels dans le corpus les termes «multi-modal», «interobjets», «multivalué» ou «multi-agents», il est tentant d'analyser les matrices $\mathbf{N} \mathbf{X}$-adj et $\mathbf{N} \mathbf{X}-\mathbf{N}$, respectivement $\mathbf{N}$ adj et $\mathbf{N} \mathbf{N}$.

Les quatre autres termes, langage $C$, moteur 0 , niveau méta et arbre "min-max", contiennent des éléments disparates, difficiles à classer. «Meta» est un formant quelque peu productif, mais ici, il n'a pas cette fonction; par ailleurs, il n'est pas suffisamment lexicalisé pour pouvoir être considéré adjectif ou nom. Quant à «min-max», dans le terme arbre «min-max», il s'agit d'une construction tronquée héritée de l'anglais (de «minimax procedure» [Boulanger 1988: 12]).

N ADV...

Les composés $\mathbf{N}$ adv... sont au nombre de dix, répartis sur deux matrices:

- $\mathbf{N}$ adv (3 termes) : chaînage arrière, chaînage avant, retour arrière.

- $\mathbf{N}$ adv-adj (7 termes): formule bien formée, graphe non orienté, logique non décidable, logique non monotone, raisonnement non monotone, solution sous-optimale, système non monotone.

En ce qui a trait à la seconde matrice, on constate que les termes sont tous formés à l'aide de l'adverbe «non», à l'exception de formule bien formée et solution sousoptimale; de plus, on retrouve presque toujours l'expression «non monotone», notion clé de l'intelligence artificielle et des systèmes experts. Notons que, une fois de plus, l'adverbe (sauf pour formule bien formée) s'apparente davantage à un élément formant. Ainsi, graphe orienté et raisonnement monotone sont également des termes du corpus, et 
«solution optimale», bien qu'il n'y figure pas, n'est pas aberrant, contrairement à «formule formée».

Les types de composés qui suivent sont parmi les moins productifs. ADV-N...

Les six termes issus de la forme de composition en adv-N... font appel à deux adverbes précédemment rencontrés: «non» et «sous». Un seul d'entre eux, sous-tâche experte, possède une extension (adjectivale), tandis que les cinq autres répondent à la matrice de base adv-N: non-connexité, non-monotonicité, non-monotonie, sous-arbre et sous-but. Pour ce qui est des deux derniers termes, il s'agit plutôt de dérivation préfixale.

N V...

Six termes sont créés selon le modèle $\mathbf{N}$ V... Nous obtenons quatre matrices, mais deux d'entre elles sont pour le moins artificielles; l'emploi des guillemets pour les termes qui leur correspondent confirme d'ailleurs cette impression: relation «possède» $(\mathbf{N} \mathbf{V})$ et relation «est-un» (N V-det).

Dans le cas des quatre autres termes, que se partagent deux matrices, l'élément verbal est davantage en fonction attribut. Ce sont d'abord les termes de la matrice $\mathbf{N ~ V ~ N ~}$ qui empruntent la construction «orienté objets»: langage orienté objets, programmation «orientée objets» et représentation "orientée objets» (la présence des guillemets indique ici le caractère étranger de la construction). L'autre terme, enseignement assisté par ordinateur, qui répond à la matrice $\mathbf{N} \mathbf{V}$ prep $\mathbf{N}$, est également calqué de l'anglais (de «computer-aided teaching» [Pavel 1986: 3]).

N CONJ...

Les composés $\mathbf{N}$ conj... sont parmi les plus marginaux du corpus. Ils ont été analysés au pied de la lettre; en fait, si leurs composants n'avaient pas été identifiables, ils auraient été classés dans une catégorie «autres».

Ainsi, on obtient deux matrices pour trois termes très formels: $\mathbf{N}$ conj adv pour règle si...alors, puis $\mathbf{N}$ conj conj pour arbre «ET-OU» et graphe ET/OU. Les deux derniers termes sont à rapprocher de ceux de la matrice $\mathbf{N}$ N-N (ex.: analyse finsmoyens), qui ont été analysés $\mathbf{N} \mathbf{N}$.

\section{SIGLAISON}

Le corpus contient 18 sigles; il a déjà été question de certains d'entre eux précédemment: IA (intelligence artificielle), SE (système expert), CAC (capacité d'atteindre toute conclusion) et $C D C$ (capacité de discriminer parmi tout ensemble de conclusions possibles).

Parmi cet ensemble de sigles, on retrouve trois variantes qui illustrent bien le type de variation graphique lié à ce procédé de formation. En premier lieu, la séparation ou non des majuscules initiales par des points: IA en concurrence avec I.A., P.O.O. (programmation «orientée objets») ou POO. L'abandon de la ponctuation est généralement fonction de l'intégration du sigle dans l'usage des utilisateurs. Il existe donc au départ un certain flottement orthographique.

Le second type de variation, qui correspond à la dernière étape d'intégration du sigle, est l'abandon des majuscules: PROLOG (programmation logique), qui devient Prolog. Le terme n'est plus senti comme abréviation et est susceptible d'engendrer des dérivés ou des composés («prologien» [Pavel 1988: 2], «machine Prolog» [Colmerauer 1987: 286]). 
Le terme Prolog est d'ailleurs un excellent exemple de sigle prononçable en un mot ou «acronyme»; ici, comme c'est fréquemment le cas, l'acronyme est composé des premières lettres de chaque mot, plutôt que des seules initiales. Cependant, il n'est pas certain que les acronymes potentiels du corpus soient prononcés comme des mots. Ainsi, $S E$ se prononce plutôt «ès-se» que «se», IA se prononce habituellement en faisant une pause entre les deux voyelles, tandis que EAO (enseignement assisté par ordinateur) est imprononçable s'il n'est pas épelé.

En revanche, $L O O$ (langage orienté objets) et $P O O$ sont prononçables en un mot (bien que le résultat soit bizarre), de même que S.I.A.D. (système interactif d'aide à la décision), CAC et t.a.d. (type abstrait de données). Notons que le dernier terme ainsi que c.v. (coefficient de vraisemblance) sont en minuscules, ce qui montre qu'il s'agit davantage d'abréviations que de sigles. $C A C$ et $C D C$ seraient également à classer avec les abréviations.

Par ailleurs, aucun des sigles du corpus n'inclut les mots-outils des termes de départ: $A G C$ (atelier de génie cognitif), $B C$ (base de connaissances), $B F$ (base de faits), $S C$ (source de connaissances), EAO (enseignement assisté par ordinateur), S.I.A.D. (système interactif d'aide à la décision) etc.; la question ne se pose pas lorsque le terme de départ lui-même ne possède pas de mots-outils: système expert, langage orienté objets, etc.

Somme toute, la composition occupe une place prépondérante dans le corpus, et par extension, dans l'intégralité du technolecte des systèmes experts (Pavel 1986: 4). Les matrices terminogéniques les plus productives sont les suivantes:

Tableau 3. Matrices les plus productives

\begin{tabular}{|r|l|c|c|}
\hline & MATRICE & NOMBRE & \% (Composés) \\
\hline $\mathbf{1}$ & N adj & 141 & 29,4 \\
\hline $\mathbf{2}$ & N prep N & 128 & 26,7 \\
\hline $\mathbf{3}$ & N N & 29 & 6,1 \\
\hline $\mathbf{4}$ & Sigles & 18 & 3,8 \\
\hline $\mathbf{5}$ & N Prep N adj & 17 & 3,5 \\
\hline $\mathbf{6}$ & N prep N prep N & 15 & 3,1 \\
\hline & N prep det N & 15 & 3,1 \\
\hline $\mathbf{7}$ & X-N & 13 & 2,7 \\
\hline $\mathbf{8}$ & N adj adj & 9 & 1,9 \\
\hline $\mathbf{9}$ & N adv (-) adj & 7 & 1,5 \\
\hline & N prep (N prep det N) & 7 & 1,5 \\
\hline $\mathbf{1 0}$ & N N-N & 5 & 1 \\
\hline & N prep NX & 5 & 1 \\
\hline & N prep adj N & 5 & $\mathbf{1}$ \\
\hline & adv-N & 5 & 1 \\
\hline & & 419 & $87 \%$ \\
\hline
\end{tabular}


Aucune de ces matrices n'est à rejeter, lorsque l'on prend en considération la composition au second degré. Par ailleurs, 20 matrices sont touchées par ce procédé (sur un grand total de 50), bien que seulement 49 termes sur 479 (en excluant ceux de la matrice X-N, davantage assimilables aux dérivés), en soient issus. Sur ces 20 matrices, 11 seraient à rejeter, puisque les termes qu'elles génèrent seraient redistribués dans les listes d'autres matrices plus productives. En définitive, si l'on ajoute à ces 11 matrices celles du type «....N-N»: N conj conj (arbre «ET-OU»), N N-N (procédé alpha-bêta, processus producteur-consommateur), $\mathbf{N}$ prep $\mathbf{N}-\mathbf{N}$ (opération d'unification-instanciation) ou $\mathbf{N} \mathbf{X}$ $\mathbf{X}$ (arbre «min-max»), ainsi que toutes celles qui ne diffèrent de la matrice principale que par la présence d'un déterminant, $\mathbf{N}$ prep $\mathbf{N}$ prep det $\mathbf{N}, \mathbf{N}$ prep $\mathbf{N}$ prep det $\mathbf{N}$ prep $\mathbf{N}, \mathbf{N}$ prep det $\mathbf{N}$ et $\mathbf{N}$ prep det $\mathbf{N}$ prep $\mathbf{N}$, ce sont 19 matrices qui devraient être éliminées, portant à 31 le nombre de matrices réellement génératrices de termes dans le technolecte français des systèmes experts.

Il est à noter que tous les composés du corpus sont formés à partir d'un ou plusieurs noms; font apparemment exception les sigles et abréviations mais, bien entendu, pour chacun d'eux, le terme de départ contient au moins un nom. Compte tenu de l'importance de l'élément nominal au sein des composés, il serait intéressant de caractériser la productivité des termes selon le nombre de noms qu'ils comportent ( $c f$. Portelance 1986:2).

Ainsi, 198 multitermes ( $41 \%$ du total) sont mononominaux (fait initial, graphe non orienté, arbre «ET-OU», langage $C$, sous-arbre, IA, etc.); 231 termes, soit $48 \%$ des composés (tout près de la moitié), sont binominaux (tri binaire par arbre, ingénieur cogniticien, noud d'entrée, système à tableau noir, moteur d'ordre 2, capacité d'atteindre toute conclusion, langage orienté objets, système multi-agents, t.a.d. (de type abstrait de données), etc.); 43 multitermes contiennent trois noms (réseau sémantique à propagation de marqueurs, processus producteur-consommateur, programmation à base d'objets, machine à inférence à architectures parallèles et intégrées, outil de maintien de la cohérence, $C D C$ [de capacité de discriminer parmi tout ensemble de conclusions possibles], etc.); enfin, seulement sept termes sont quadrinominaux (cycle de base du moteur d'inférence, cycle d'acquisition et de rafraîchissement des données, validation en temps réel et à pied d'euvre, etc.). Aucun terme ne possède plus de quatre noms.

Signalons encore un procédé relevé en quelques occasions, l'ellipse, c'est-à-dire la suppression d'un des éléments d'un terme, ce dernier devenant sous-entendu (sur le modèle de «[voiture] automobile»). Ainsi, moteur d'inférences nu génère moteur nu élémentaire, où «d'inférences» est absent, mais implicite. De la même façon, machine parallèle sous-entend la notion d'architecture parallèle, telle qu'elle est exprimée dans le terme machine à inférence à architectures parallèles et intégrées. Langage objets et programmation objet proviennent tous deux de la construction «...orienté objets», «orienté» étant omis. Quant à moteur 0 , il s'agit de la version tronquée de moteur d'ordre 0 .

Deux termes sont à cet égard intéressants: système de production, issu du croisement de système expert et règle de production; d'ailleurs, le terme non tronqué (système expert à règles de production) apparaît également dans le corpus. Le deuxième terme est système de résolution, où système expert est une fois de plus tronqué, mais ici, contrairement au cas précédent, le deuxième composant provient de l'ellipse de l'élément final du terme, et non de l'élément initial: résolution (de problèmes).

Plusieurs termes débutant par «système» devraient théoriquement être pourvus de l'adjectif «expert», particulièrement ceux de la série «système d'aide à»: système d'aide à la prescription médicale est un raccourci pour «système expert d'aide à la prescription médicale». Toutefois, système multi-experts s'accommoderait mal de l'insertion de l'adjectif «expert», bien que celui-ci soit effectivement sous-entendu. Précisons cependant que système à base de connaissances ne fait pas automatiquement référence à 
un système expert. Bien qu'il s'agisse de la même technologie, un système est véritablement expert lorsque sa base de connaissances contient sous forme codée le savoir d'un expert (Pavel 1986: 3).

Inversement, on retrouve des cas de surspécification, tels que raisonnement monotone, qui se retrouve également sous la forme raisonnement de type monotone, dans le corpus. Il ne peut être ici question de troncation, puisque «de type» est pour ainsi dire, vide de sens.

\section{DÉRIVATION}

Il a été dit précédemment que 135 termes, soit $21,6 \%$ du corpus, étaient des monotermes. Cependant, si l'équation «multiterme $=$ composé» est vraie dans la majorité des cas, l'équation «monoterme = dérivé» se vérifie beaucoup plus rarement.

Dans les faits, la grande majorité des monotermes sont issus d'emprunts à d'autres technolectes, notamment à ceux de l'informatique traditionnelle (algorithme, compilateur, interface, etc.) et de la logique (induction, prédicat, quantificateur, tautologie, etc.). Sinon, ce sont des mots ou termes d'usage général: apprentissage, conclusion, expert, maquette, parent, etc. Employés dans le domaine des systèmes experts, ces termes acquièrent un sens particulier, mais, en aucun cas, il ne s'agit de dérivés.

Si l'on considère uniquement les néologismes formels, on constate qu'ils sont peu nombreux. Ainsi, seuls intellectique, robotique, cognitique, cogniticien (et sa variante cognitien) sont des créations dues à une dérivation. Le suffixe «-ique» signifie ici 'application de l'informatique à...' (Taylor 1988 : 552). Robotique a été obtenu par simple suffixation (《robot» et «-ique»), tandis que cognitique est à classer parmi les motsvalises, de «cognit[if]» et «[informat]ique», bien que de façon plus probable, la genèse de ce terme soit ingénieur de la connaissance $\rightarrow$ ingénieur cogniticien (par dérivation et apposition) $\rightarrow$ cogniticien (par ellipse) $\rightarrow$ cognitique (par dérivation et analogie avec les termes en «-ique»). Quant à intellectique, c'est une création difficile à cerner puisque, par définition, elle désigne une discipline réunissant les sciences cognitives, le génie cognitif et la représentation des connaissances (Pavel 1989a: 173). On pourrait affirmer qu'il s'agit d'un mot-valise constitué par «intelligence» et «informatique».

De manière moins rigide, il est possible d'ajouter à ces dérivés purs un certain nombre de dérivés non néologiques, dont les bases sont des termes-clés du domaine des systèmes experts. Il s'agirait alors de néologie de sens, à défaut de néologie formelle. Ainsi, on retrouve expertise (de expert), formalisation (de «formel»), interfaçage et interfacer (de interface), maquettage (de maquette), modélisation et modéliser (de modèle), modularité (de module), planificateur (de plan d'action), procédural (de procédure), prototypage (de prototype), structuration (de structure), etc. De fait, certains de ces dérivés, tels interfacer, maquettage, modéliser, modularité et prototypage, ne figurent pas dans tous les dictionnaires du français.

En revanche, il semble que ce soit au sein des multitermes que se rencontrent les principaux cas de dérivation. La question de la préfixation a auparavant été traitée lors de l'étude de certaines matrices terminogéniques. Rappelons que les formants les plus productifs sont «méta» (métaraisonnement, méta-classe, etc.), «multi» (logique multivalente, système multi-agents, etc.), et «pré» (précondition, préordre, présystème), puis les adverbes «non» (non-connexité, non-monotonie, etc.) et «sous» (solution sousoptimale, sous-arbre, etc.). Quant à «hyper» (hyperarc, hypergraphe), «inter» (héritage inter-objets) et «mi» (mi-ordre), ils sont improductifs. Aux termes déjà cités dans le texte, on peut encore ajouter recherche bidirectionnelle, stratégie irrévocable, contexte de redémarrage et composant logiciel réutilisable. 
En ce qui a trait à la suffixation, on constate la productivité de plusieurs suffixes: -tion: acquisition de connaissances, affectation sylvestre, algorithme d'unification, configuration de validation, espace de résolution, filtrage par propagation, génération de plans, planification hiérarchique, système d'aide à la conception, test de continuation, etc.

-eur: capteur d'acquisition des données, démonstrateur de théorèmes, descripteur de type, générateur de plans d'action, interpréteur (de faits et de règles), pointeur, résolveur général de problèmes, unificateur, etc.

-el (-iel, -uel): branchement conditionnel, calcul formel, connaissance assertionnelle, construction manuelle, opérateur intentionnel, parcours partiel, programmation textuelle, recherche bidirectionnelle, système essentiel, etc.

-é : enseignement assisté par ordinateur, fait structuré, logique multivaluée, machine à intelligence spécialisée, mode interprété, recherche ordonnée, variable typée, etc.

-ique: apprentissage automatique, cognitique, formule atomique, information symbolique, parallélisme macroscopique, raisonnement hypothétique, schéma générique, etc.

-age: chaînage arrière, contexte de redémarrage, filtrage par propagation, héritage de propriétés, interfaçage, module d'apprentissage, outil de maquettage, prototypage, etc.

-ment: appariement, attachement procédural, branchement conditionnel, condition de déclenchement, cycle d'acquisition et de rafraîchissement des données, fonction d'ordonnancement, règle de détachement, etc.

-if: connaissance déclarative, connaissance descriptive, génie cognitif, machine déductive, prototype évolutif, raisonnement déductif, etc.

-(c)ité : arité, capacité d'atteindre toute conclusion, fonctionnalité objet, modularité, nonmonotonicité, rationalité limitée, etc.

-ie: génie cognitif, ingénierie de la connaissance, non-monotonie, raisonnement par analogie, stratégie de contrôle, etc.

D'autres suffixes, fréquents en langue générale, sont peu représentés ici : «-able» (composant logiciel réutilisable, logique non décidable, prototype jetable, stratégie irrévocable), «-ien» (cogniticien, cognitien, système expert diagnosticien, module d'aide au maintien de la cohérence), «-isme» (parallélisme macroscopique), «-iste» (base ensembliste, système déterministe).

Pour sa part, la paire «-ance/-ant» (cafficient de vraisemblance, mise en correspondance, valeur de confiance, niveau structurant, noud pendant, etc.) est peu productive, tout comme la paire «-ence/-ent» (occurrence, inférence, loi de précédence, formule logique cohérente, logique multivalente, machine intelligente, recherche arborescente, etc.).

Enfin, quelques autres formants sont à tout le moins marginaux : «-et» (triplet valué), «-ible» (noud accessible), «-ise» (prise d'expertise, mise à jour de connaissances), «-itude» (facteur de certitude), «-oire» (connaissance opératoire), «-ule» (formule bien formée, module d'apprentissage) et «-ure» (architecture, procédure).

La dérivation régressive est pour ainsi dire inexistante, à l'exception peut-être du terme tri binaire par arbre. Toutefois, la dérivation impropre est un peu plus fréquente, bien qu'elle touche peu d'unités distinctes: heuristique (information heuristique, règle heuristique, etc.), logique (inférence logique, programmation logique, etc.), «logiciel» (génie logiciel, architecture logicielle, etc.), expert (interface expert, module expert, etc.), sans omettre traitement de l'incertain et raisonnement par l'absurde, déjà cités. Seuls 
deux termes formés par dérivation parasynthétique ont été relevés: appariement et encapsulation.

Terminons sur un procédé déjà énoncé par Pavel (1986: 3), qui met en évidence l'importance du suffixe «-eur» dans certaines constructions: l'alternance «résolution/résolveur» dans système de résolution/résolveur général de problèmes. D'une manière analogue, citons aussi programme de démonstration de théorèmes/démonstrateur de théorèmes, générateur de plans d'action/planificateur, et mécanisme d'interprétation/ interpréteur. De même, on retrouve une alternance des suffixes «-tion» et «-if» dans certains termes: déduction/raisonnement déductif, induction/raisonnement inductif. Enfin, notons l'opposition raisonnement analogiquefraisonnement par analogie.

\section{EMPRUNTS}

Sont ici réunis 11 termes issus d'une langue étrangère, qui ne possèdent pas d'équivalent en français, ou, s'il existe, est jugé peu satisfaisant ou est tout simplement moins employé que le terme original. Nous n'aborderons pas ici la question de l'emprunt sémantique, préférant mettre l'accent sur les emprunts de forme.

Deux termes de logique sont empruntés au latin: modus ponens et modus tollens. Tous les autres termes sont des emprunts au technolecte anglais de l'intelligence artificielle. Ainsi, on trouve plusieurs sigles et acronymes: FDL (frame description language), lips (logical inferences per second), 'inférences logiques par seconde', unité de mesure des systèmes d'IA, et LISP (ou Lisp) (list processing language), langage de programmation traitant les listes. Puis divers termes, avec ou sans équivalent français: blackboard, qui entre dans la formation de termes du type modèle de blackboard, et ce, en dépit du fait que tableau noir a été proposé comme équivalent; frame problem, calqué problème du cadre de référence, ou parfois, «problème du décor» (dans Pavel 1989a: 403); pattern-matching, qui continue à être employé, malgré les équivalents filtrage et appariement, jugés adéquats par les utilisateurs; script, qui est toujours en usage, en concurrence avec scénario; enfin tuple, terme propre aux bases de données relationnelles, qui est un emprunt intégral, sans équivalent français.

Ont été exclues ici les multiples appellations de systèmes experts, d'outils de développement et de moteurs d'inférences (MYCIN, ELITHO, MDX, etc.), puisque d'une part, ce ne sont pas réellement des termes et, d'autre part, ils sont généralement formés à partir de l'anglais, ce qui déborde de notre propos. Précisons que les termes examinés plus haut ont été retenus dans le but d'illustrer le flottement terminologique, fréquent lorsqu'un technolecte est tributaire d'une technologie d'abord développée aux États-Unis.

\section{CONCLUSION}

Bien que la composition (simple ou au second degré) occupe la majeure partie du corpus, il a été démontré que la dérivation, de façon beaucoup plus subtile, est elle aussi très présente. En confrontant nos données avec celles qui ont été recueillies par Pavel (1989b: 347), on note plusieurs similitudes: 1) primauté des suffixes «-tion» et «-eur» (et à un moindre niveau «-age») dans la formation des noms d'agent ou d'action; 2) faiblesse de «-itude» et «-isme» par rapport à «-ité» pour les noms dérivés d'adjectifs; 3) dérivation impropre, principalement pour les termes en «-ique» et «-iel» (suffixes par ailleurs plutôt productifs); et 4) rareté des mots-valises, fait surprenant pour le français, qui en fait habituellement grand usage. Toutefois, la préfixation est peu importante, et seuls quelques formants («méta», «multi», «non» et «sous») sont quelque peu productifs.

La quasi-totalité des termes du corpus sont nominaux (99\%), puisque seulement quatre adjectifs et deux verbes ont été relevés, sur un total de 625 termes. De plus, les 
données ayant trait au nombre de noms dans les composés sont en accord avec Portelance (1986: 2): abondance de binominaux en comparaison des tri et quadrinominaux (due en grande partie à la matrice $\mathbf{N}$ prep $\mathbf{N}$ ). Comme on le voit, bien que nos résultats soient fragmentaires, ils viennent confirmer ceux des précédentes études. En outre, il serait intéressant de comparer les présents modèles avec les modèles propres à d'autres technolectes, qu'ils soient ou non hors du domaine de l'intelligence artificielle.

* Recherche réalisée grâce à une subvention du Centre francophone de recherche en informatisation des organisations et du Conseil de recherches en sciences humaines du Canada.

\section{BIBLIOGRAPHIE}

BOULANGER, Jean-Claude (1987): «Le miroir aux alouettes en intelligence artificielle», Meta, 32-3, pp. 326-331.

BOULANGER, Jean-Claude (1988): «Un miroir aux alouettes: le calque en intelligence artificielle», Le langage et l'homme, 23-66, pp. 3-13.

CLAS, André (1988): «Une matrice terminogénétique en plein essor: les binominaux juxtaposés», Textlinguistik und Fachsprache. Reiner Arntz (éd.), AILA-Symposions, Hildesheim, 13-16 avril 1987, Hildesheim, Zürich, New York, Georg Olms Verlag, pp. 215-227.

COIFFET, Philippe (1986): «Vocabulaire de la productique». La Banque des mots, 32, pp. 187-204.

COLMERAUER, Alain (1987): «Prolog, langage de l'intelligence artificielle», La Recherche en intelligence artificielle, articles choisis et présentés par Pierre Vandeginste, Paris, Seuil, pp. 285-312.

GEHENOT, Daniel (1975) : «Le sigle - aperçu linguistique», Meta, 20-4, pp. 271 -307.

KOOI, Rens (1989) : «Vocabulaire de la traduction automatique», La Banque des mots, 37, pp. 11-35.

LETHUILLIER, Jacques et Monique C. CORMIER (1990): «Terminologie français-anglais des systèmes experts et des sujets connexes», Meta, 35-4.

LURQUIN, Georges (1982): «INTER et META dans le vocabulaire scientifique et technique contemporain», L'Actualité terminologique, 15-1, pp. 1-2.

OTMAN, Gabriel (1988): «Trois décennies d'intelligence artificielle», La Banque des mots, Numéro spécial, pp. 41-46.

F'AVEL, Silvia (1986): «Some Aspects of the Terminology of Artificial Intelligence (Part One)», L'Actualité terminologique, 19-8, pp. 1-4.

PAVEL, Silvia (1987): «Vocabulaire contextuel de l'intelligence artificielle», La Banque des mots, 33, pp. 67-90.

PAVEL, Silvia (1988): «Siglaison et créativité lexicale en intelligence artificielle», L'Actualité terminologique, $21-4$, pp. $1-4$

PAVEL, Silvia (1989a): Intelligence logicielle. Dictionnaire français-anglais, Ottawa, Réseau international de néologie et de teminologie, Module canadien, Ministre des Approvisionnements et Services Canada, xii $+503 \mathrm{p}$

PAVEL, Silvia (1989b): «Niveaux linguistiques et terminologie de l'intelligence artificielle», Meta, 34-3, pp. 344-351.

PORTELANCE, Christine (1986): «À propos du rôle des matrices terminogéniques dans le développement des langues de spécialités», Terminogramme, 39-40, pp. 1-3.

QUIRION, Jean (1989): Vocabulaire anglais-français des systèmes experts en conception assistée par ordinateur, Essai pour l'obtention du grade de maîtrise en terminologie et traduction, Québec, Université Laval, 167 p. (non publié).

TAYLOR, Glen (1988): «INFOR-M-A-T-IQUE: A Study of «informatique»-related «ique» Terms», Meta, 33-4, pp. 550-560. 


\section{ANNEXE 1: CORPUS}

\section{ANALYSÉ}

A

acquisition de connaissances acquisition des connaissances adresse

affectation sylvestre

AGC (atelier de génie cognitif)

aide à la prise de décision algorithme

algorithme alpha-bêta algorithme d'unification analyse fins-moyens ancêtre

appariement

application

apprentissage

apprentissage automatique

arborescence

arborescence binaire

arborescence de règles

arbre

arbre binaire

arbre de raisonnement

arbre des états

arbre «ET-OU»

arbre «min-max»

arc

architecture

architecture hybride

architecture logicielle

architecture parallèle

arité

aspect

assertion

atelier de génie cognitif

atelier de génie logiciel

atome

attachement procédural

attribut

attribut propre

axiome

\section{B}

base de connaissances

base de données

base de données déductive

base de faits

base de règles

base ensembliste

$\mathrm{BC}$ (base de connaissances)

$\mathrm{BF}$ (base de faits)

[blackboard]

branchement conditionnel but
C

CAC (capacité d'atteindre toute conclusion)

calcul des prédicats

calcul des propositions

calcul formel

calcul parallèle

capacité d'atteindre toute conclusion

capacité de discriminer parmi tout ensemble de conclusions possibles

capteur d'acquisition des données

case

CDC (capacité de discriminer parmi tout ensemble de conclusions possibles)

cellule

chaînage arrière

chaînage avant

chaînage en arrière

chaînage en avant

chaînage mixte

chaîne de raisonnement

chemin

chemin courant

classe

classe élémentaire

clause

cafficient de vraisemblance

cogniticien

cogniticien de développement

cogniticien d'exploitation

cognitien

cognitique

combinatoire

commentaire

compilateur

compilation de «réseaux de programmes

composant logiciel réutilisable

concept intermédiaire

conclusion

condition

condition d'arrêt

condition de déclenchement

configuration de validation

connaissance assertionnelle

connaissance de bon sens

connaissance déclarative

connaissance de contrôle

connaissance de définition

connaissance descriptive

connaissance experte

connaissance opératoire

connaissance procédurale construction incrémentale

construction manuelle

contexte de redémarrage

contradiction

contrainte

coquille

c.v. (coefficient de

vraisemblance

cybernétique

cycle d'acquisition et de

rafraîchissement des données

cycle de base du moteur

d'inférence

cycle du moteur d'inférences

\section{D}

déclaratif

déclencheur (d'une règle)

déduction

défaut

délégation

démon

démonstrateur

démonstrateur de théorèmes

descendant

descripteur

descripteur de type

diagnostic

diagnostic ponctue

documentation

document de la connaissance

donnée

E

EAO (enseignement assisté diteur

encapsulation

enseignement assisté par

ordinateur

ensemble de conflit

envoi de message

espace de recherche

espace de résolution

espace des états

espace des solutions

espace d'états

état

exemplaire

expert

expertise

explication du raisonnement

exploration d'arbre

explosion combinatoire

expression logique 


\section{F}

facette

facteur de certitude

fait

fait à valeurs multiples

fait binaire

fait imprécis

fait incertain

fait initial

fait structure

[FDL] [frame description language]

feuille

filtrage

filtrage par propagation

fils

fonction

fonction d'évaluation

fonction d'ordonnancement

fonction membre

fonctionnalité objet

formalisation

formalisation des

connaissances

forme-objet

forme propositionnelle

formule atomique

formule bien formée

formule logique cohérente

formule logique composée

fragment de connaissance

fragment de savoir

[frame problem]

\section{G}

généralisation

généralisation existentielle

générateur de plans d'action

générateur de systèmes experts

génération de plans

génic logiciel

graphe

graphe de l'espace des états

graphe de recherche

graphe de relations

graphe des états

graphe ET/OU

graphe non orienté

graphe orienté

graphe simple

graphe valué

\section{$\mathbf{H}$}

héritage

héritage de propriétés

héritage de spécialisation

héritage hiérarchique

héritage inter-objets

héritage multiple

heuristique hyperarc

hypergraphe

hypothèse du monde clos

hypothèse du monde fermé

hypothèse du monde ouvert

IA (intelligence artificielle)

I.A.

îlot de confiance

induction

inférence

inférence logique

information atomique

information dynamique

information heuristique

information statique

information symbolique

ingénierie de la connaissance

ingénieur cogniticien

ingénieur de la connaissance

ingénieur en intelligence artificielle

ingénieur IA

instance

instanciation

intégration symbolique

intellectique

intelligence artificielle

interfaçage

interface

interface de consultation

interface de développement

interface expert

interfacer

interface utilisateur

interprète

interpréteur

interpréteur (de faits et de

$$
\text { règles) }
$$

interpréteur de règles

intervalle

itération

J

jeu d'essai

K

k-connecteur

\section{L}

langage acteurs

langage $\mathrm{C}$

langage naturel

langage objets

langage orienté objets

langage symbolique

lips

Lisp

LISP liste

logiciel d'aide à la décision

logiciel de développement de systèmes experts

logique

logique à plusieurs valeurs

logique de l'incertain

logique des prédicats

logique des propositions

logique d'ordre 0

logique du premier ordre

logique épistémique

logique floue

logique modale

logique multivalente

logique multi-valuée

logique multivaluée

logique non décidable

logique non monotone

logique temporelle

loi de précédence

LOO (langage orienté objets)

M

machine à inférence à architectures parallèles et intégrées

machine à intelligence spécialisée

machine dédiée

machine déductive

machine intelligente

machine multiprocesseurs

machine parallèle

maquettage

maquette

mécanisme de contrôle mécanisme de déduction mécanisme d'inférence mécanisme d'interprétation mémoire de travail message

méta-classe

méta-connaissance

métaconnaissance

métalangage

métaraisonnement

méta-règle

méthode

mi-ordre

mise à jour de connaissances

mise en correspondance

mode développement

mode d'inférence

mode exploitation

mode fermé

mode interprété

modèle

modèle de blackboard

modélisation

modélisation cognitive 
modéliser

mode ouvert

modification de connaissances modularité

module d'acquisition

module d'aide à la validation

module d'aide au maintien de

la cohérence

module d'aide au transfert

d'expertise

module d'apprentissage

module de contrôle

module de récupération de

l'expertise

module d'explication

module d'inférence

module expert

modus ponens

modus tollens

monotone

mot

moteur de développement

moteur d'inférence

moteur d'inférences

moteur d'inférences nu

moteur d'ordre 0

moteur d'ordre $0+$

moteur d'ordre

moteur d'ordre 2

moteur 0

moteur nu élémentaire

\section{$\mathbf{N}$}

niveau cognitif

niveau conceptuel

niveau méta

niveau structurant

noud

nœud accessible

nœud-ancêtre

noud-but

nœud de départ

nœud d'entrée

noud de raisonnement

nœud descendant

noud-feuille

noud parent

nœud pendant

nœud père

noud-racine

noud-solution

nœud terminal

non-connexité

nnon-monotonicité

non-monotonie

noyau

\section{o}

objet

objet modèle objet terminal

objet structuré

objet valué

occurrence

opérateur

opérateur de décomposition

opérateur de développement

opérateur de transformation

opérateur intentionnel

opérateur logique

opération d'unification-

instanciation

opération neutre

ordre binaire

ordre d'un système

outil d'aide à l'acquisition de connaissances

outil d'aide à la décision

outil d'aide au développement

outil de développement

outil de développement de SE

outil de développement de

systèmes experts

outil de maintien de la

cohérence

outil de maquettage

paquet de règles

parallélisme macroscopique parallélisme microscopique parcours

parcours d'un arbre

parcours partiel

parent

pas de raisonnement

[pattern-matching]

planificateur

planification hiérarchique

pointeur

POO (programmation

P.O.O.

portée d'une variable

pouvoir de conclusion

précondition

prédicat

prédicat du premier ordre

prémisse

préordre

présystème

principe de résolution

prise de décision

prise d'expertise

problème du cadre de référence

procédé alpha-bêta

procédural

procédure

procédure alpha-bêta procédure «démon»

procédure «domestique»

processeur

processeur symbolique

processus de résolution

processus producteur-

consommateur

profondeur

programmation à base d'objets

programmation automatique

programmation classique

programmation déclarative

programmation en règles de production

programmation logique

programmation objet

programmation «orientée

objets»

programmation par

apprentissage

programmation textuelle

programme de démonstration de théorèmes

Prolog

PROLOG

proposition

proposition composée

prototypage

prototype

prototype (traduction de

$$
\text { frame) }
$$

prototype de test

prototype évolutif

prototype jetable

Q

quantificateur

$\mathbf{R}$

racine

raisonncment

raisonnement analogique

raisonnement approximatif

raisonnement déductif

raisonnement de sens commun

raisonnement de type

monotone

raisonnement hypothétique

raisonnement incertain

raisonnement inductif

raisonnement monotone

raisonnement non monotone

raisonnement par analogie

raisonnement par défaut

raisonnement par l'absurde

raisonnement régressif

raisonnement temporel

rationalité limitée

recherche arborescente

recherche avec retour arrière 
recherche bidirectionnelle recherche du meilleur d'abord recherche en faisceau

recherche en largeur recherche en largeur d'abord recherche en profondeur recherche en profondeur d'abord

recherche exhaustive recherche heuristique recherche ordonnée récursion

règle

règle conditionnelle

règle de contraintes

règle de décisions

règle de défaut

règle de détachement

règle déductive

règle de production

règle d'inférence

règle floue

règle heuristique

règle imprécise

règle incertaine

règle par défaut

règle si...alors

relation «est-un(e)»

relation "possède»

représentant

représentation analogique représentation déclarative représentation de l'incertain représentation des

connaissances

représentation hybride

représentation multi-modale

représentation «orientée objets»

représentation par objets structurés

représentation par règles de production

représentation par schémas

représentation procédurale

réseau sémantique

réseau sémantique à propagation de marqueurs

résolution de conflit

résolution de problèmes

résolveur général de problèmes

restriction

retour arrière

robotique

robotique/action

robotique fondamentale

robotique industrielle

robotique/perception

rubrique
$S$

$\mathrm{SC}$ (source de connaissances) scénario

schéma (synonyme de prototype)

schéma

schéma d'objet

schéma générique

sciences cognitives

script

SE (système expert)

sélection

sémantique d'un nœud

sens d'un noud

senseur

SE temps réel

S.I.A.D. (système interactif d'aide à la décision)

situation

solution sous-optimale

sommet

source de connaissances

sous-arbre

sous-but

sous-tâche experte

spécialisation

spécialisation universelle

stratégie de contrôle

stratégie de raisonnement

stratégie de résolution

stratégie essai

stratégie essai avec retour arrière

stratégie irrévocable

structuration

structure d'accueil

successeur

suivi de poste

surcharge

syllogisme

système à base de

connaissances

système à base de

connaissances interactif

système à règles de production

système à tableau noir

système d'aide à la conception

système d'aide à la décision

système d'aide à la

prescription médicale

système d'aide à la prise de décision

système d'apprentissage

système de contraintes

système de contrôle

système de deuxième génération système de prise de décision

système de production

système de résolution système déterministe

système d'IA

système d'inférence

système d'intelligence artificielle

système essentiel

système-expert

système expert

système expert à objets

système expert à règles de production

système expert auxiliaire

système expert de deuxième génération

système expert diagnosticien

système expert industriel

système expert multi-niveaux

système expert nu

système expert temps réel

système expert universel vide

système expert vide

système général

système interactif d'aide à la décision

système multi-agents

système multi-experts

système non monotone

système temps réel

T

tableau noir

tâche experte

t.a.d. (type abstrait de

données)

tautologie

terme

test d'arrêt

test de continuation

théorie des sous-ensembles

flous

théorie logique

trace

traceur

traitement de l'incertain

traitement de l'incertitude

traitement symbolique

transfert de connaissances

transfert de l'expertise

transfert d'expertise

tri binaire par arbre

triplet valué

tuple

type

type abstrait de données

$\mathbf{U}$

unificateur

unification

utilisateur expert 
utilisateur final

utilisateur opérateur

V

valeur de confiance

valeur de défaut

valeur de vérité

valeur incertaine

valeur indéterminée

valeur multiple

valcur par défaut

valeur-possible

validation

validation en temps réel et à

pied d'œuvre

variable

variable logique

variable propositionnelle

variable typée 